\title{
Possibilité de réalisation des ouvrages de prise d'eau en bordure de mer : Problèmes se posant au concepteur hydraulicien
}

\author{
A. Le Grand \\ Bergeron S.A.
}

\section{Introduction}

Les problèmes particuliers liés au pompage de l'eau de mer ont été abordés lors de la session organisée les 18 et 19 novembre 1971 par la Société Hydrotechnique de France sur le thème: "L'Hydraulique et les industries littorales".

Dans le texte qui suit, les problèmes qui résultent de la houle sont examinés d'une façon plus détaillée, en montrant l'influence que celle-ci peut avoir sur la conception même des ouvrages de.prise d'eau.

Il est précisé que les ouvrages considérés sont relatifs à des stations de pompage de moyenne importance, soit un débit pompé dé 5 à $30000 \mathrm{~m}^{3} / \mathrm{h}$ environ.

\section{Éléments de base}

Dans le cadre d'un besoin donné d'eau de mer se situant sur un site également donné, une étude préalable complète s'avère nécessaire pour définir l"architecture hydraulique la mieux appropriée à partir de laquelle les ouvrages seront réalisés.

Force est tout d'abord de constater que les conditions techniques particulièrement favorables pour !'implantation d'une prise d'eau de mer ne sont jämais au nombre des criteres de choix d'un site.

Dès lors l'architecture hydraulique la mieux appropriée est celle qui, tout en s'accomodant des difficultés locales dues à la houle, conduit au coût le plus réduit de réalisation et d'exploitation à fiabilité égale.

Les éléments de base à prendre en compte pour la conception générale de l'installation sont :
- Pour les houles au large :

- leur direction

- leur amplitude avec probabilités d'avènement et de dépassement

- la fréquence des tempêtes et ‘du gros temps

- la durée des périodes d'accalmie et les époques correspondantes

- Pour la côte :

- son orientation générale par rapport aux houles

- son relief (falaise, dune)

- sa nature (rocheuse, alluviale, sablonneuse).

- Pour les fonds :

- les emplacements les plus proches présentant un tirant d'eau suffisant (carte des fonds)

- la nature des fonds.

Un littoral " difficile», qui nous serviira de base pour notre examen, sera avant tout caractérisé par :

- une houle quasi permanente, formant une barre à proximité plus ou moins immédiate de la côte;

- une côte basse aveć fonds sablonneux sur un substratum rocheux.

Une telle cote se rencontre couramment, par exemple sur le littoral atlantique des continents européen et africain (cf. fig. 1).

Des cas connus de réalisation ont été mentionnés lors de la session précitée organisée par la Société Hydrotechnique de France, à savoir:

- prise d'eau du complexe chimique de production d'engrais installée à Safi par la Société Krebs pour le compte de Maroc-Chimie;

- prise d'éau de l'usine de la Société Industrielle d'En- 
grais du Sénégal installée à M'Bao par la Société d'Engineering Compadec;

- prise d'eau de Seignosse sur la côte Aquitaine étudiée par SOGREAH.

Ils vont servir de base à nos réflexions.

\section{Commentaires sur des exemples de réalisation}

Dans les trois exemples précités de réalisation on peut remarquer que :

- Pour la prise d'eau de Maroc-Chimie à Safi, située sur une côte particulièrement inhospitalière, une étude systématique des fonds et du relief sous-marin a permis de déceler un étroit chenal naturel aboutissant dans une excavation au pied de la falaise.

L'ensemble de l'ouvrage de prise a donc pu être réalisé à l'abri d'une paroi rocheuse naturelle formant bouchon.

Ce dernier a été supprimé, le moment venu, à l'explosif, pour faire communiquer le chenal naturel avec le bassin anti-houle.

La conception de l'ensemble des ouvrages de prise d'eau et de pompage a donc été entièrement conditionnée par la présence de ce bouchon rocheux, à l'abri duquel il était possible de travailler malgré des brisants quasi permanents.

Il s'agit là d'un cas très exceptionnel.

- Pour la prise d'eau de la S.I.E.S. à M'Bao, la côte est uniformément sablonneuse. L'existence de périodes d'accalmie suffisamment longues a permis d'exécuter un canal d'amenée entre deux bajoyers en palplanches, fermé à son extrémité amont, par la tête de prise d'eau. Celle-ci est constituée par trois caissons préfabriqués formant bassin anti-houle, assemblés rigidement entre eux, reliés au canal et protégés contre la houle par un épis massif en palplanches et en béton coulé sous l'eau.

Dans ce cas particulier, la conception de l'ouvrage a donc été conditionnée par l'existence des périodes d'accalmie sans brisants.

- Pour la prise d'eau de Seignosse étudiée par SOGREAH, sur la cote Aquitaine, les fonds sont de nature sablonneuse - comme dans l'exemple de M'Bao qui précède - mais il n'y a pas de période d'accalmie de houle.

La nature du sous-sol permettant d'envisager des pieux battus, le concepteur a choisi la solution d'une estacade pour atteindre les fonds présentant le tirant d'eau suffisant pour la prise d'eau.

Il est intéressant de noter que la solution consistant à lancer et ensouiller une conduite sur la plage avait également été étudiée par SOGREah. Elle a été éliminée du fait des difficultés de mise en place.

Le débit pompé étant relativement réduit $\left(400 \mathrm{~m}^{3} / \mathrm{h}\right)$ SOGREAH a pu loger des pompes immergées en tête d'ouvrage ce qui, comme nous le verrons, n'est en général pas retenu pour des débits peu importants.

Par conséquent, la conduite sur estacade étant en charge, la longueur de l'estacade et sa hauteur par rapport à la mer sont sans grande influence sur le pompage.
- Mais pourquoi à M'Bao, par exemple, ne pas avoir installé une estacade comme à Seignosse?

Si. à Seignosse, l'importance de la houle et des vagues déferlantes excluaient toute solution en canal ouvert, à M'Bao il aurait été concevable de remplacer le canal par une estacade.

Pour un même problème, on est donc en présence de deux conceptions entièrement différentes du point de vue hydraulique, tant pour lamortissement de la houle que pour les dépôts de sable, la protection contre les algues et coquillages, etc.

Ce choix devient donc affaires de prix et de moyens matériels disponibles pour réaliser l'ouvrage.

Ces différents exemples illustrent une partie de l'éventail des solutions possibles, caractérisées par la distance séparant le littoral non immergé (ou découvrant à marée basse) de la zone en mer où le tirant d'eau est suffisant pour alimenter l'installation :

- Si la distance est très faible. la prise d'eau peut être exécutée à l'abri d'un bouchon qui peut être soit rocheux naturel (ex. : Safi), soit créé artificiellement.

- Si la distance n'est pas faible, la prise d'eau à concevoir comprend:

- d'une part un ourrage de tête situé au large;

- dautre part un ouvrage de liaison entre l'ouvrage de tête et les installations à terre.

Le problème est alors de :

- choisir le type de prise d'eau en fonction des données hydrauliques et des moyens disponibles pour la réalisation:

- choisir l'emplacement le mieux adapté

\section{Choix du type de prise d'eau}

L'hypothèse de départ est que la prise d'eau est fortement exposée aux effets destructeurs de la houle. Comme les réalisations industrielles considérées nécessitent des équipements électromécaniques de filtration et de pompage d'une certaine importance, l'étude est conduite de préférence avec implantation de ces équipements à terre. S'il ne peut en être ainsi, principalement pour des questions d'ensablement de la prise d'eau comme nous le verrons par la suite, leur implantation doit être faite dans une tour en mer.

Il s’agit alors d'un véritable ouvrage maritime dont le coût est toujours relativement élevé.

Dans le présent chapitre, nous n'allons donc considérer que le cas le plus fréquent des équipements à terre, c'est-à-dire que l'ouvrage de liaison travaille en aspiration.

On peut tout d'abord classer les prises' d'eau en deux familles en fonction de la nature de l'écoulement dans l'ouvrage de liaison.

Celui-ci peut être en effet :

- soit à surface libre : la prise d'eau est alors en canal;

- soit en conduites:

- en siphon si l'aqueduc est sous vide,

- immergée dans le cas contraire. 


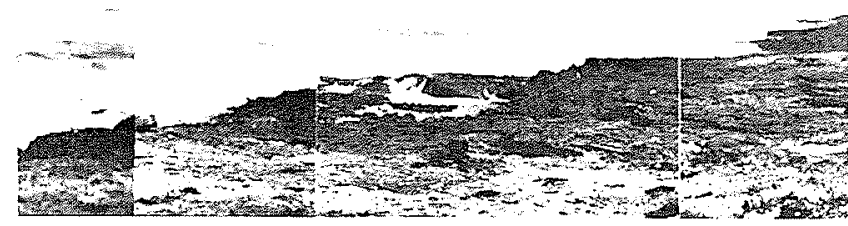

$1 /$ Mohammedia (Maroc)

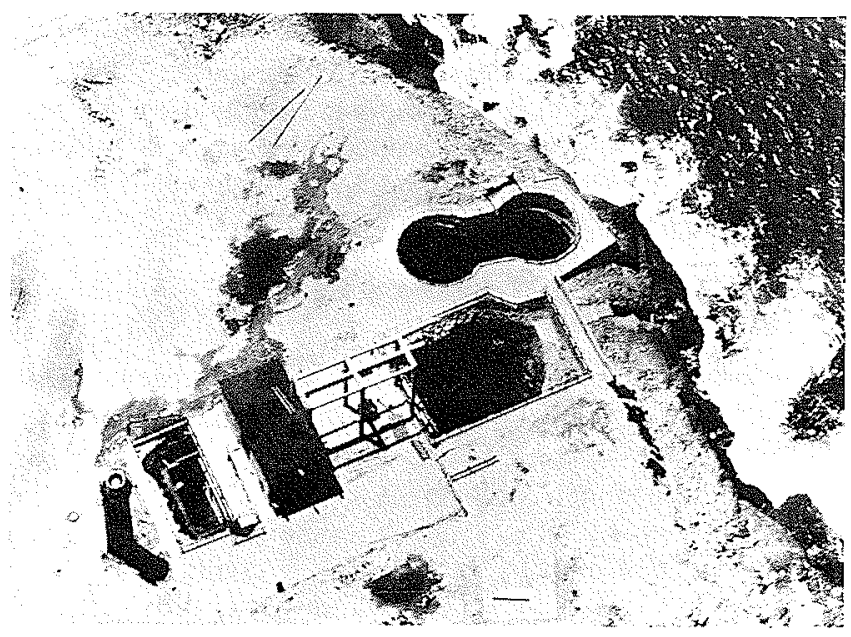

2 / Prise d'eau de Maroc-Chimle

\section{1 - SOLUTION « CANAL »}

La solution “ canal » est, en général, considérée par les entrepreneurs comme moins coûteuse de réalisation que la solution « conduite".

L'ouvrage comprend alors deux digues latérales, de longueur suffisante pour que le tirant d'eau minimum soit atteint en toute sécurité à marée basse.

Ces digues sont exécutées à l'avancement par des engins terrestres.

Elles peuvent être, soit en enrochements (prise d'eau de la centrale thermique du cap des Biches au Sénégal), soit en palplanches (prise d'eau de la S.I.E.S. au Sénégal).

La digue en enrochements paraît recevoir la préférence.

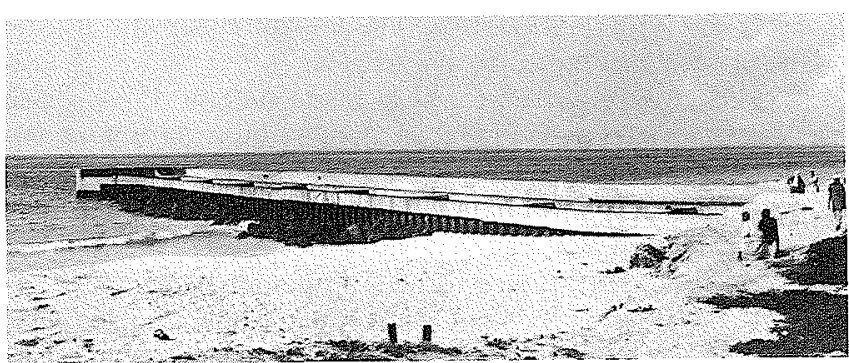

3 , Prise d'eau de la S.I.E.S. à M'Bao (République du Sénégal)

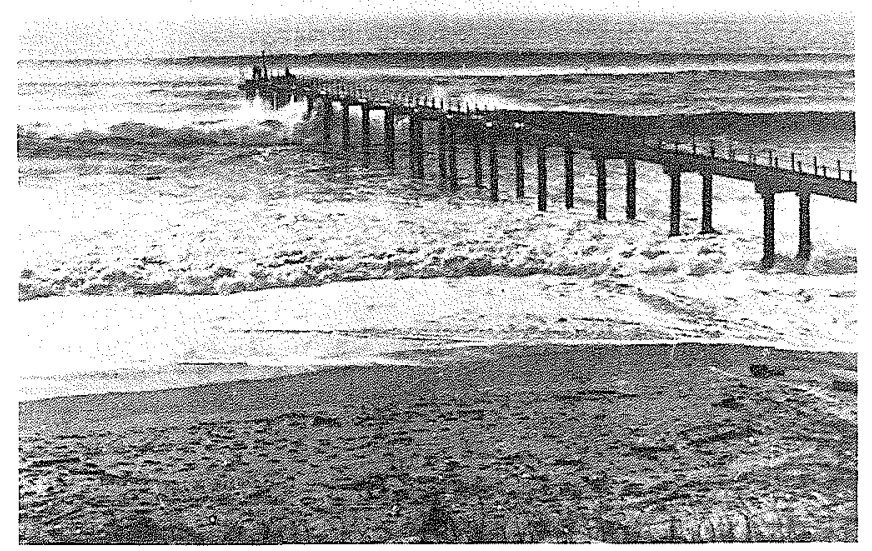

4 / Prlse d'eau de Selgnosse (Cliché SOGREAH)
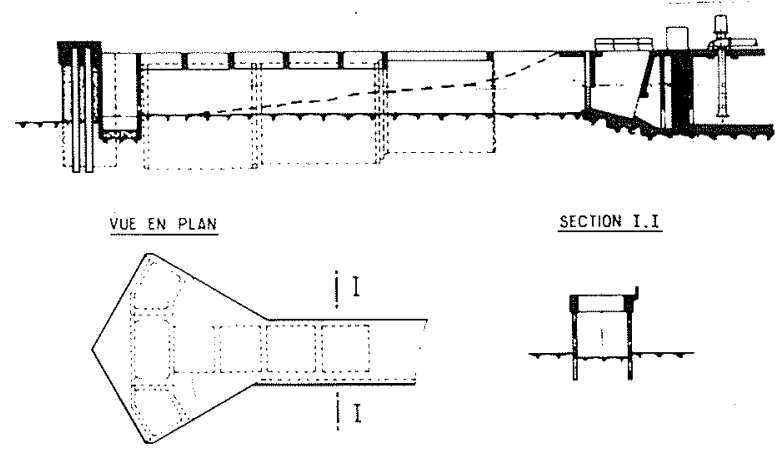

SECTION I.I

5 / Canal en palplanches 
Ceci est vrai si l'ouvrage est implanté sur un littoral relativement peu battu par les vagues.

Il peut en être autrement si la houle déferle d'une façon permanente sur ce littoral (car alors la mise en ceuvre devient plus difficile) et si, lors des tempêtes, l'ouvrage est soumis aux multiples effets destructeurs des brisants.

La protection des bajoyers du canal et de l'ouvrage de tête contre les différentes formes de houles déferlantes nécessite alors des enrochements de plus en plus importants. ce qui finit par en augmenter le coût d'une façon inconsidérée.

Bien entendu, cette solution sous-entend que des enrochements de dimension convenable sont disponibles à des conditions intéressantes dans l'arrière-pays (la région dunkerquoise, par exemple, est considérée comme peu propice à cet égard).

Lorsque les conditions énumérées ci-dessus ne sont pas rassemblées. il peut être alors intéressant d'exécuter les digues en palplanches.

Le canal ainsi obtenu se termine en mer par un ouvrage de tête, soit en enrochement, soit en maçonnerie.

Cet ouvrage doit être conçu hydrauliquement pour amortir les amplitudes de la houle dans le canal - en général par "résistance", c'est-à-dire par des ouvertures de sections judicieusement calculées.

Il doit être aussi conçu mécaniquement pour résister sans dommage aux effets de la houle à laquelle il se trouve exposé de plein fouet, ce qui conduit nécessairement à un ouvrage très massif.

Il faut noter que la solution "canal " présente l'inconvénient de modifier le profil du littoral en constituant un épi par rapport à celui-ci. Sous l'effet de courants parallèles à la côte, entraînant une interruption du transport littoral, il se forme des dépôts de sable, de part et d'autre de l'épi, qui peuvent finir par obstruer l'ouvrage de tète.

En dernier lieu, le canal constituant une zone à faible vitesse d'écoulement, des dépôts de sable peuvent rapidement se produire nécessitant alors la mise en place d'un pont dessableur.

Lorsque le marnage dû à la marée est important. ce phénomène est accentué à chaque marée haute.

En résumé, la solution "canal " peut être intéressante toutes les fois que le littoral présente les caractéristiques suivantes :

- faible marnage et déclivité assez forte (afin de ne pas être conduit à des dimensions trop importantes en longueur et en hauteur pour les digues latérales):

- zone naturellement protégée des tempêtes et des déferlements de houles qui les accompagnent:

- période daccalmie de quelques mois par an pour faciliter la mise en cuvre;

- faible teneur en sable dans l'eau par gros temps.

S’il n'en est pas ainsi et que, malgré cela, la solution canal est retenue, un pont dévaseur s'impose avec tous les inconvénients qu’un tel engin entraîne.

Cette solution convient donc principalement pour les cas faciles.

\section{2 - SOLUTION «CONDUITE»}

Jusqu'à présent, nous n'avons retenu que le cas de l'implantation à terre des équipements de filtration et de pom-

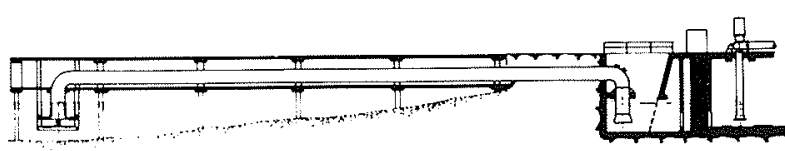

\section{6 / Siphon sur estacade}

page. Par voie de conséquence. l'ouvrage de tête est réduit à un simple entonnement immergé à la profondeur voulue, et pouvant comporter des grilles à barreaux largement espacés pour narréter que les corps dont les dimensions ne seraient pas compatibles avec celles des conduites.

Le probleme it traiter est donc celui de la traversée de la zone des brisants par l'ouvrage de liaison en conduite. La conduite est an siphon sous vide (puisque la pompe est à terre) si la traversée est au-dessus de la mer, elle est immergée dans le cas contraire.

Pour les débits moyens qui nous intéressent, les diamètres des conduites sont couramment compris entre $500 \mathrm{~mm}$ et $1000 \mathrm{~mm}$. cest-it-dire que ces conduites ne sont pas ou sont difficilement visitables. ce qui est un inconvénient.

Par contre. lavantage par rapport au canal est de pouvoir maintenir des vitesses compatibles avec la mise en suspension des sables el si possible. suffisamment grandes pour éviter les proliférations de coquillages.

\section{1 - Conduite en siphon}

Du point de vue hydraulique. le siphon présente deux avantages extrêmement intéressants par rapport aux autres solutions, at savoir:

- Dans un siphon a larrét. il n'y a pas lieu de craindre une proliferation d'algues ou de coquillages. Il suffit, en effet. de le désamoreer. De plus. et à condition que son diametre soit superieur it $600 \mathrm{~mm}$. il devient visitable, ce qui permel de le nentoger et de lentretenir au moindre coût.

- Si la structure qui le supporte est ajourée (estacade), sa présence ne modifie en aucune façon l'environnement sous-marin. cest-à-dire que, dans le cas d'une côte sablonneuse. louvrage ne peut lui-même être la cause d'un ensablement supplémentaire dans le temps.

Comme. de plus. les circuits et les équipements de mise sous vide et dentretien du vide. s`ils sont bien conçus, sont parfaitement sûrs. cette solution est finalement la plus fiable au niveau de lexploitation et la plus aisée au niveau de lentretien.

Elle est donc conseillée toutes les fois que le site le permet.

\section{Ses limites peuvent résulter:}

- Dun marnage trop important. Il est en effet souhaitable que le siphon soit au-dessus des plus hautes crêtes de houle pour ne pas etre soumis a des efforts mécaniques prejudiciables.

Dans ces conditions. un vide de 6 at $7 \mathrm{~m}$ doit être considéré comme limite supérieure à ne pas dépasser pour ne pas rencontrer des problemes importants de dégazage et surlout pour qu il ne puisse jamais $y$ avoir cavitation dans la conduite lors des régimes transitoires provoqués par la houle. 


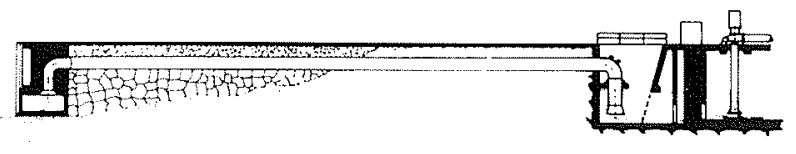

7 / slphon sur digue en enrochements

- D'une côte en pente douce (1 à $2 \%$ ).

Le vide dans le siphon, au point le plus défavorisé, est évidemment fonction de la perte de charge dans celui-ci, donc de la longueur de l'ouvrage.

Une longueur importante, qui peut être nécessaire pour atteindre l'immersion voulue au droit de l'entonnement, peut devenir incompatible avec la condition du vide maximum mentionnée ci-dessus ( 6 à $7 \mathrm{~m}$ ).

- D'une zone très exposée aux tempêtes excluant tout ouvrage en superstructure.

Du point de vue supportage, le siphon peut reposer sur une digue (en enrochements ou en palplanches) ou sur une estacade (sur pieux battus ou forés).

Le choix entre ces diverses solutions est affaire de nature du sous-sol (substratum rocheux ou non), de dimensionnement de l'ouvrage pour résister au déferlement de la houle et de moyens localement disponibles pour réaliser l'ouvrage.

C'est donc le coût qui peut départager.

\section{2. - Conduite immergée}

Par rapport aux solutions précédemment envisagées (canal, siphon), celle de la conduite immergée présente de nombreux inconvénients. C'est pourquoi elle n'est envisagée que lorsque les deux autres sont exclues.

Ces inconvénients sont les suivants :

- difficulté de mise en cuvre:

- difficulté d’ancrage:

- visites et nettoyages le plus souvent impossibles posant, par voie de conséquence, le problème de la pérennité de l'ouvrage:

- possibilité d'ensablement de la prise de tête du fait de la modification de l'environnement sous-marin;

- risque d'apparition de régime de dépôt de sable, principalement si des marches à débit réduit sont envisagées.

Pour y remédier, ceci peut même conduire à accepter de déverser à l'arrivée le surplus de débit non consommé, de telle sorte que le débit pompé ne soit jamais inférieur à la valeur minimum acceptable fixée par le régime de dépôt.

Au point de vue installation, les conduites immergées peuvent être :

- soit ensouillées dans le sable;

- soit ancrées dans le fond d'une tranchée creusée dans le rocher;

- soit incorporées à l'intérieur d'une digue exécutée à l'avancement.

Comme l'ouvrage considéré est supposé se situer dans une zone où la houle déferle, de nouveaux problèmes doivent être évoqués:

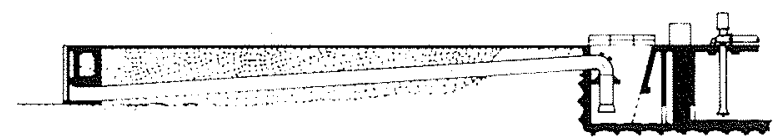

$8 /$ Condulte Immergée sous enrochements

- Pour les conduites ensouillées dans le sable, la question de la profondeur de l'ensouillement doit être étudiée très attentivement afin que les conduites ne soient pas mises à découvert lors de fortes tempêtes.

- Si une période daccalmie de deux ou trois mois par an n'existe pas, l'ensouillement ou l'ancrage peuvent se concevoir à l'abri d'un port de travaux, à condition toutefois que la longueur de l'ouvrage ne dépasse pas une cinquantaine de mètres.

La réalisation de cet ouvrage provisoire est en effet nécessairement coûteuse.

Si les conditions ci-dessus ne sont pas remplies, deux solutions se présentent :

- soit incorporer la conduite à l'intérieur d'une digue à l'avancement;

- soit poser la conduite entre deux digues parallèles et la bloquer avec des enrochements (entre les deux digues).

Le choix entre ces différentes solutions est fonction des diamètres considérés pour les conduites, de la nature du sous-sol et des moyens matériels localement disponibles.

C'est finalement à nouveau la comparaison des coûts qui permettra de choisir.

\section{Choix de l'emplacement}

Ce choix demande un examen préliminaire minutieux de la bande de littoral sur laquelle l'implantation de la prise d'eau est envisagée. L'étude doit être faite par beau et mauvais temps et en plusieurs périodes de l'année (dont en particulier durant les grandes marées) de façon à bien se rendre compte des possibilités offertes par le rivage et des difficultés à surmonter par suite du déferlement de la houle.

De nombreux points sont à examiner du fait de l'utilisation de l'eau de mer. Pour ces points, il y a intérêt à se reporter à la communication faite par M. Guiton lors de la session précitée de la S.H.F. sur les usines littorales.

En plus de ces investigations habituelles, il y a lieu de s'intéresser plus particulièrement aux détails suivants:

- points de déferlement de la houle pour différentes marées;

- nature des fonds et nature des rochers:

- carte des fonds marins;

- données topographiques et géologiques concernant le terrain immédiatement en arrière du littoral;

- existence de carrières de rocher à proximité;

- examen détaillé des zones les plus abritées;

- failles ou crevasses éventuelles dans la roche en place; 
- possibilités d'accès au site par des engins terrestres ou maritimes;

- opinion des pêcheurs locaux.

Le concours d'hommes-grenouilles est toujours très profitable pour recueillir des informations supplémentaires sur tel emplacement apparemment intéressant.

Ils peuvent en effet procéder rapidement à un relevé détaillé des fonds en cet endroit (cote des fonds par rapport au niveau de la mer, nature des fonds, présence de bancs de sable, turbidité de l'eau, échantillons de sables, présence d'algues et de coquillages).

Une fois obtenues, ces informations doivent permettre de sélectionner un ou plusieurs emplacements qui pourront être jugés les plus satisfaisants car présentant les caractéristiques les plus proches de celle du site idéal à savoir :

- crique la mieux protégée des houles prépondérantes. donc convenablement orientée par rapport à celles-ci;

- absence de bancs de sable à proximité immédiate;

- forte déclivité du fond permettant d'obtenir, pour un tirant d'eau minimum donné, une prise d'eau relativement courte.

C'est à partir de là que commence la deuxième phase de l'étude qui est celle de l'estimation des coûts.

\section{Classification des solutions}

L'analyse précédente montre que les principaux facteurs locaux naturels qui pèsent le plus lors du choix d'un type de prise d'eau sont

- la nature des fonds sous-marins qui peut être sablonneuse et/ou rocheuse:

- la déclivité des fonds sous-marins qui peut être forte ou faible - en ce sens qu'elle influe directement sur la longueur de l'ouvrage de prise d'eau;

- la possibilité d'utiliser ou non des enrochements pour l'exécution de l'ouvrage.

En fonction de ces facteurs, il apparaît dès lors possible de proposer une classification des différents types de prise d'eau dont nous présentons, ci-dessous, une liste récapitulative :

- canal avec digues en enrochements;

- canal avec digues en palplanches;

- siphon sur estacade;

- siphon sur digue en enrochements;

- conduite immergée en souille;

- conduite immergée sous digue.

Cette classification ne prétend pas être limitative.

Elle n'a pour but que de faciliter une première sélection parmi les six solutions examinées ci-dessus.

Elle montre, de plus, qu'à partir du moment où les caractéristiques principales d'un site sont connues, le choix reste finalement à faire entre deux si ce n'est trois possibilités (mis à part un cas particulier).

Comme, de plus, ces caractéristiques se présentent généralement d'une façon moins tranchée que ne le fait apparaître le tableau dans un but de simplification, le choix se complique d'autant.

\section{Solutions exceptionnelles}

Pour certains cas particulièrement difficiles, il se peut qu'aucune des solutions envisagées ci-dessus ne puisse convenir.

Il en est ainsi si des aléas d'exploitation subsistent au niveau de la prise d'eau projetée, alors même que le pompage est considéré comme indispensable au fonctionnement de l'unité industrielle qu'il alimente (raffinerie, complexes sidérurgiques, chimiques, etc...)

La prise d'eau travaillant gravitairement entre le plan d'eau en mer et celui dans l'ouvrage de pompage, la charge disponible est obligatoirement réduite.

L'augmentation de la perte de charge peut donc conduire rapidement à une impossibilité pour la prise d'eau d'assurer le débit demandé par les pompes.

Ceci peut se produire, sous l'effet d'un ensablement intempestif de l'ouvrage, par apparition soit principalement d'un bouchon de sable en tête (canal ou conduite immergé), soit éventuellement d'un régime de dépôt en conduite.

Si le phénomène d'ensablement n'est que progressif, des mesures peuvent être prises pour y pallier (dragage, chasse) mais sous l'effet de tempêtes, il peut être brutal et mettre en cause le fonctionnement même du circuit.

Pour y remédier, il faut augmenter la charge disponible qui assure le débit afin que le bouchon ne puisse se former en tête d'ouvrage et que la vitesse d'écoulement reste supérieure à la vitesse limite de dépôt du sable.

Ceci exclut la solution "canal ", car sa pente est forcément limitée, aussi bien que la solution « siphon", car le

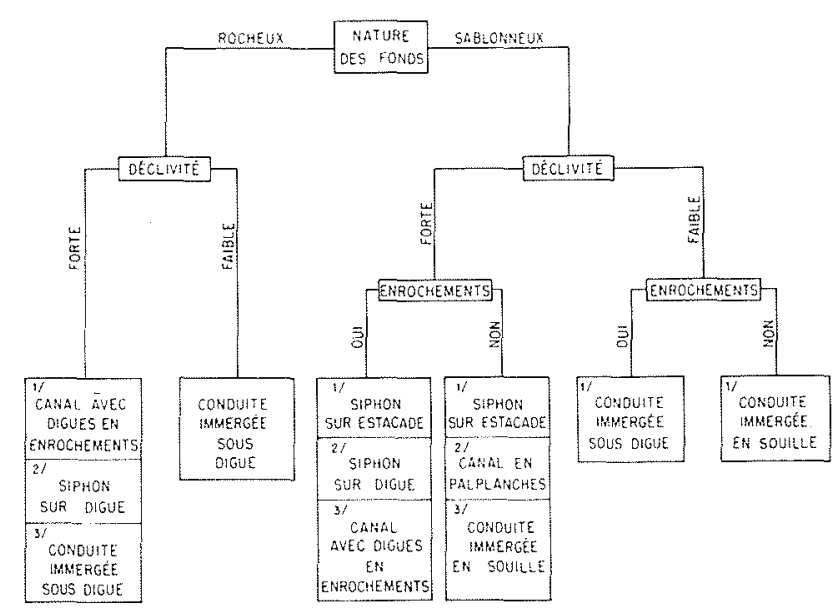

9 / Classification des solutions

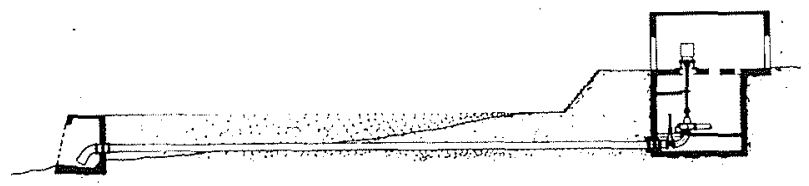

10 / Condulte immergée en aspirations directe 
seuil de la pression absolue minimum acceptable est trop rapidement atteint.

Deux solutions exceptionnelles sont alors envisageables :

\section{Solution " dragage "}

Elle consiste à raccorder l'aspiration des pompes à terre directement à une conduite immergée, de telle façon que la charge disponible entre le plan d'eau en mer et les pompes incorpore la capacité d’aspiration de celle-ci.

L'ensemble doit être alors assimilé hydrauliquement et mécaniquement à une pompe à draguer avec son élinde et son suçon, et se fonder sur les mêmes techniques de réalisation.

\section{Solution " tour en mer "}

Dans le cas précédent. la prise d'eau travaille toujours en aspiration.

La longueur de l'ouvrage en limite toutefois le champ dapplication du fait de laugmentation des pertes de charge avec la longueur de l'ouvrage et la vitesse d'écoulement recherchée.

La solution de la "tour en mer " apparait alors en dernier recours et répond techniquement aux cas les plus difficiles.

Dans cette solution, les pompes d'exhaure sont installées dans la "tour en mer". Elles aspirent directement en mer, donc sans perte de charge d'aspiration autre que celle due a l'ouvrage daspiration.

Bien entendu, ces pompes doivent être conçues mécaniquement comme de véritables pompes à draguer.

La perte de charge dans l'ouvrage de liaison entre la tour en mer et les ouvrages à terre est prise en compte dans la pression de refoulement de la pompe, ce qui permet de choisir sans difficulté les caractéristiques de cet ouvrage: qui peut être soit en siphon, soit en conduite immergée quelle que soit sa longueur.

\section{Estimation des coûts}

Une classification des techniques de réalisation des ouvrages n'a pu être abordée dans le présent cadre.

Ces techniques sont en effet fonction des engins et de lexperience dont disposent les entreprises de travaux publics.

Ces deux éléments variant beaucoup d'un pays à un autre, force est d'adapter la conception aux moyens disponibles, ce qui peut conduire à éliminer, dans tel pays, une solution qui pourra être choisie dans tel autre.

La seule étude technique de conception et de réalisation de l'ouvrage ne permettant donc pas d'apporter une réponse unique, il est nécessaire de compléter l'étude technique par une comparaison des coûts.

Le fait que l'ouvrage soit implanté dans une zone où la houle déferle, accentue limportance de la connaissance de ces coûts puisque la conception et la réalisation de l'ouvrage en sont rendues d'autant plus difficiles.

Pour chacune des solutions envisagées, les coûts doivent comprendre :

- les études complémentaires éventuelles dont, en particulier, les essais sur modèle réduit jugés nécessaires;

- les travaux de réalisation;

- la capitalisation de l'énergie consommée, dans le cas où les pertes de charge seraient très différentes d'une solution à une autre:

- la capitalisation des charges annuelles d'entretien s'il en est de même.

A partir de ce stade, le maître d'ouvrage disposera de tous les éléments pour prendre sa décision étant entendu qu'il devra faire intervenir au préalable un dernier élément non quantifiable : la fiabilité du système.

Les différentes solutions retenues pourront donc être classées en fonction de leur degré de fiabilité.

Compte tenu de l'importance donnée à ce critère, en égard au service attendu pour cette partie de l'installation, le maitre d'ouvrage pourra alors estimer les risques et prendre sa décision finale.

\section{Conclusion}

L'implantation d'une prise d'eau de mer de moyenne importance dans une zone difficile où la houle déferle, ne peut être décidée qu'au vu d'une étude approfondie traduisant les points de vue du concepteur hydraulicien et de l'entrepreneur de travaux publics.

L'hydraulicien seul, en méconnaissant des techniques de réalisation ou en sous-estimant leurs difficultés peut être conduit à proposer des solutions trop coûteuses ou même irréalisables.

Il lui appartient donc d'examiner l'éventail des solutions possibles et de s'appuyer sur les connaissances de l'entrepreneur pour les classer en fonction des coûts.

Un tel projet est donc le fruit d'une collaboration très étroite entre ces deux spécialistes.

Le maitre d'ouvrage, à qui revient le choix de la solution finale, devra, pour sa part, être conscient des difficultés du problème qui le concerne, donc de l'importance de la durée et du coût des études préliminaires qui l'aideront à prendre sa décision. 


\section{Discussion}

Présidem : M. P. BERGERON

Je remercie M. LE GRAND, dit M. le Président, pour son exposé qui pose maints problèmes pratiques; il devrait permettre une classification des différents procédés de réalisation disponibles pour y faire face. J'ouvre, sans plus tarder, la discussion sur les différents cas évoqués.

Vous n'avez pas parlé, observe M. Millot, des prises d'eau en rivage dans les zones de déferlement. Parfois, on peut être tenté, surtout sur les côtes atlantiques, où les zones de déferlement se trouvent à 400 ou $500 \mathrm{~m}$ au large de faire un pompage dans les zones rocheuses du rivage en prévoyant un bassin de tranquillisation. Dans de tels cas, on doit faire face au problème du sable entraîné en suspension; on peut, soit installer un dessableur, soit laisser transiter le sable à travers les pompes et les échangeurs en utilisant des matériaux appropriés, tels que le titane. Avez-vous abordé ce type de recherches ou d'études?

Nous avons étudié, répond $M$. LE GRAND, de tels problèmes. Dans ce cas, les solutions que nous avons préconisées correspondent à un pompage en mer, pour éviter les phénomènes de dépôt de sable dans la conduite d'amenée. Le matériel de pompage peut aisément être conçu pour véhiculer du sable (il existe des pompes à draguer) mais certains équipements qui se trouvent en aval ne peuvent s'en accomoder : on peut aussi rencontrer des difficultés d'exploitation en ce qui concerne la filtration par exemple; il ne faut pas que le bassin de filtration devienne un bassin de décantation. Si cette éventualité est à craindre, il faut établir un bassin de dessablement avant la filtration et le dégrillage. On revient ainsi au schéma d'une station d'exhaure équipée de pompes spéciales, d'une digue en mer, d'une longue conduite passant en dessous de la zone de déferlement de houle pour arriver au bassin de dessablage, lequel va être suivi par la filtration et un nouveau pompage de reprise. En général, il nous est demandé de ne pas faire transiter de sable dans les circuits d'utilisation.

Vous n'avez pas cité, dit M. BeAudesson (G.E.M., Hersent), les prises d'eau constituées par une conduite sous-marine supportée par des pieux moulés ou coulés; cette solution a été utilisée pour des émissaires en mer (Rio de Janeiro, notamment) mais peut-être pas pour des prises d'eau.

En effet, répond $M$. LE GRAND, c'est une technique de réalisation qui n'a pas été mentionnée; elle s'apparente à celle d'une conduite ensouillée ou ancrée dans le rocher avec des encoches venant caler la tuyauterie.

M. le Président clôt la discussion en remerciant tous ceux qui l'ont animée. Il donne ensuite la parole à M. BARBIER pour l'exposé de sa communication. 